DYNAMICAL SYSTEMS AND ERGODIC THEORY BANACH CENTER PUBLICATIONS, VOLUME 23 PWN - POLISH SCIENTIFIC PUBLISHERS WARSZAWA 1989

\title{
ERGODIC AND CENTRAL LIMIT THEOREMS IN STATISTICAL MECHANICS IN CONTINUOUS CASE
}

\author{
KRYSTYNA PARCZYK \\ Institute of Mathematics, Nicholas Copernicus University \\ Torun, Poland
}

\section{Introduction}

It is known that a physical quantity on the $n$-particle phase-space is defined as a real functions. But such a definition of physical quantity cannot be transferred into the phase-space of locally finite configurations of unlabelled particles in $\boldsymbol{R}^{d}$. In $[1,2]$ the author has proposed a definition of a physical quantity on the space mentioned above. In mathematical statistical mechanics literature some simple species of such physical quantities are studied: point random fields for which central limit theorems are examined (see references in [3]), and Lanford's observables for which some limit theorems have been proved [4], including space-average theorem.

In this article all results for a whole class of measurable physical quantities are summarized, namely, phase-average, space-average and timeaverage theorems. Also an independent physical quantity is defined. For some independent physical quantities such as point random fields and interaction potential with null boundary condition central limit theorems are known for some class of Gibbs measures (see references in [3]). In this article one untypical result [3], which deals with central limit theorem for point random fields, is quoted. This central limit theorem is true even for some nonergodic Gibbs measures.

\section{Fundamental definitions [5]}

Phase space $Y$ is defined as the set of locally finite configurations $x \in \mathscr{Y}$ of unlabelled particles $(q, p) \in \boldsymbol{R}^{d} \times \boldsymbol{R}^{d}$, which are described by their position $q \in \boldsymbol{R}^{d}$ and momentum $p \in \boldsymbol{R}^{d}$.

The "locally finite configuration $x$ " means that the configuration $x$ has only a finite number of particles in any bounded region $V \subset \dot{R}^{a}$ : 
Card $x_{V}<\infty$, where $x_{V}=\{(q, p) \in x, q \in V\}, V \subset R^{d}$ is bounded.

Additionally it is assumed that in any one position can be only one particle:

if $\left(q, p_{1}\right),\left(q, p_{2}\right) \in x$ then $p_{1}=p_{2}$.

The phase space $\mathscr{Y}$ is equipped with the natural topology and the $\sigma$-algebra of Borel sets. The space-translation group $\boldsymbol{R}^{d}$ acts on $\mathscr{x}$ by translating each position of a particle by a vector $r \in R^{d}$ :

$$
x+r=\left\{(q+r, p):(q, p) \in x_{\}}, \quad r \in \mathbb{R}^{d} .\right.
$$

Gibbs measure $\mu$. For the interaction potential $U$ and parameters $\beta>0$, $\bar{\mu} \in R^{1}$, a Gibbs measure $\mu$ is defined as a probability measure satisfying the equilibrium equations:

$$
\begin{aligned}
& \text { for any } f \in L^{1}(\mathscr{Y}, \mu) \text { and any bounded Borel set } V \subset R^{d} \\
& \int_{i} f d \mu=\sum_{n=1}^{\infty} \frac{1}{n !} \int_{,\left(V^{c}\right)} \mu(d y) \underset{V^{n} \times \mathbb{R}^{d n}}{\int} d q_{1} \ldots d q_{n} d p_{1} \ldots d p_{n} \\
& \exp \left\{-\beta\left[-\bar{\mu} n+\sum_{1}^{n} p_{i}^{2} / 2+U\left(q_{1}, \ldots, q_{n} \mid y\right)\right]\right\} f\left(\left\{\left(q_{1}, p_{1}\right), \ldots,\left(q_{n}, p_{n}\right)\right\} \cup y\right) \\
& +\int_{x\left(V^{c}\right)} \mu(d y) f(y)
\end{aligned}
$$

where $\mathscr{Y}(W)=\left\{x_{W} ; x \in \mathscr{Y}\right\}$ is the subspace of locally finite configurations of unlabelled particles in $W \subset \boldsymbol{R}^{d}$.

We will assume that the interaction potential $U$ and parameters $\beta, \bar{\mu}$ are such that a Gibbs measure exists.

\section{Physical quantities (observables) $[1,2]$}

From the viewpoint of ergodic theorem we are interested in physical quantities which depend on particles. On the phase space $\mathscr{X}$ we do not enumerate particles but we can distinguish a finite set of particles in a fixed bounded region $V \subset \boldsymbol{R}^{d}$. Thus physical quantities may be defined as certain systems of real functions

$$
f(\cdot, V): \mathscr{X} \rightarrow \boldsymbol{R}^{1},
$$

where $V$ runs over the bounded Borel subsets of $\boldsymbol{R}^{d}$.

For configuration $x \in \mathscr{X}$ the value $f(x, V)$ is interpreted as the physical quantity for the finite set $x_{V}$ of particles from $x$ situated in $V$, including all their interactions with particles outside $V$. This interpretation imposes the following definition: 
A physical quantity, hence forth abbreviated $p q$, is a system $\{f(\cdot, V)\}$ of functions $f(\cdot, V): \mathscr{Y} \rightarrow \boldsymbol{R}^{1}$, where $V$ runs over bounded Borel subsets of $\boldsymbol{R}^{d}$, satisfying

(i) $f(x, \emptyset)=0$,

(ii) if $x_{V}=x_{W}$ then $f(x, V)=f(x, W)$,

(iii) $f(\cdot, V): \mathscr{H} \rightarrow \boldsymbol{R}^{1}$ are measurable.

EXAMPLES OF $p q$ 's:

Ex 1. The number of particles: $f(x, V)=\operatorname{Card} x_{V}$.

Ex 2. Potential energy:

$$
f(x, V)=U\left(x_{V} \mid x_{V c}\right)=\underset{\operatorname{def}}{=} \lim _{\uparrow^{c}}\left[U\left(x_{V \cup W}\right)-U\left(x_{W}\right)\right] .
$$

Ex 3. The potential energy with null boundary conditions:

$$
f(x, V)=U\left(x_{V}\right) \text {. }
$$

Ex 4. Kinetic energy: $f(x, V)=\frac{1}{2} \sum_{1}^{n} p_{i}^{2}$ if $x_{V}=\left\{\left(q_{1}, p_{1}\right), \ldots,\left(q_{n}, p_{n}\right)\right\}$.

Ex 5. Free path of particles:

$$
f(x, V)=\sum_{(q, p) \in x, q \in V} f(q, x),
$$

where $f(q, x)$ is the free path of the particle $(q, p) \in x$, i.e. $f(q, x)$ is the distance which the particle $(q, p)$ covers to the first colision with another particle from $x$.

The definition of $p q$ has physical interpretation but is impractical in technical use. A $p q$ can be defined equivalently as a measurable real function on another space, which is in some sense built over the phase space $\mathscr{X}$. This space $\mathscr{Y}$ is defined by

$$
\mathscr{Y}=\bigcup_{\emptyset \neq A \text { tinite } \subset \boldsymbol{R}^{d}} A \times \mathscr{Y}_{A},
$$

where

$$
\mathscr{X}_{A}=\{x \in \mathscr{X}: \pi x \supset A\} \text {, }
$$

$\pi x=$ set of positions of particles of $x$, (i.e. $\mathscr{X}_{A}=$ the subset of these configurations $x \in \mathscr{H}$ which have particles positioned in each point of $A$ ).

The topology on $\mathscr{Y}$ is defined by the conditions of convergence:

$$
\left(A_{n}, x_{n}\right) \rightarrow(A, x) \text { in } \mathscr{Y} \Leftrightarrow\left\{\begin{array}{l}
x_{n} \rightarrow x \text { in } \mathscr{X}, \\
A_{n} \rightarrow A \text { in relative topology on } \pi \mathscr{X} .
\end{array}\right.
$$

The space $\mathscr{Y}$ is equipped with the $\sigma$-algebra of Borel sets. 
The equivalence between a real Borel function $f: \mathscr{Y} \rightarrow R^{1}$ and a system of functions $\{f(\cdot, V)\}$ satisfying conditions (i)-(iii) is established by

$$
f(y)=\sum_{B \subset A}(-1)^{\operatorname{Card}(A \backslash B)} f(x, B), \quad \text { where } y=(A, x) \in \mathscr{Y},
$$

and by

$$
f(x, V)=\sum_{y=(A, x) \in \psi^{*}, A \subset V} f(y) .
$$

For the above examples and for $y=(A, x)$ :

Ex 1.

$$
f(y)= \begin{cases}1 & \text { if Card } A=1, \\ 0 & \text { otherwise. }\end{cases}
$$

Ex 2. If the interaction potential $U$ is associated with a pair potential $\varphi$, i.e.

$$
U(x, V)=\frac{1}{2} \sum_{\substack{q \neq q_{1}, q, q_{1} \in \pi x_{V}}} \varphi\left(q-q_{1}\right)+\sum_{\substack{q \in \pi x_{V}, q_{1} \in \pi x_{V c}}} \varphi\left(q-q_{1}\right),
$$

then

$$
U(y)= \begin{cases}\sum_{\left.q_{1} \in \pi x \backslash q\right\}} \varphi\left(q-q_{1}\right) & \text { if } A=\{q\}, \\ -\varphi\left(q-q_{1}\right) & \text { if } A=\left\{q, q_{1}\right\} \\ 0 & \text { otherwise. }\end{cases}
$$

Ex 3. If the interaction potential $U$ is associated with a pair-potential $\varphi$, then for a potential energy $f$ with null boundary condition

$$
f(y)= \begin{cases}\varphi\left(q-q_{1}\right) & \text { if } A=\left\{q, q_{1}\right\} \\ 0 & \text { otherwise }\end{cases}
$$

Ex 4. Kinetic energy

$$
f(y)= \begin{cases}p^{2} / 2 & \text { if } A=\{q\} \text { and }(q, p) \in x, \\ 0 & \text { otherwise }\end{cases}
$$

Ex 5. Free path of particles

$$
f(y)= \begin{cases}\text { free path for }(q, p) \in x, & \text { if } A=\{q\}, \\ 0 & \text { otherwise }\end{cases}
$$

The space-translation group $\boldsymbol{R}^{d}$ acts on $\mathscr{Y}$ according to the formula

$$
(A, x)+r=(A+r, x+r) \text {. }
$$


The physical quantity $f: y \rightarrow \boldsymbol{R}^{\mathbf{1}}$, which is translation invariant, i.e.

$$
f(A, x)=f(A+r, x+r), \quad r \in \boldsymbol{R}^{d},
$$

may be treated as a real Borel function on the quotient space $y / \boldsymbol{R}^{d}$.

For every translation-invariant . Gibbs measure $\mu$ on the phase space $: x$ we can define a $\sigma$-finite Borel measure $v$ on the quotient space $\mathscr{Y} / \boldsymbol{R}^{d}$. First we define a probability Borel measure $\mu_{A}$ on the subspace $\mathscr{X}_{A}, A \subset \boldsymbol{R}^{d}$ finite, by the formula

$$
\begin{aligned}
\mu_{\left\{q_{0}, \ldots, q_{n}\right\}}(d x)=\varrho\left(q_{0}, \ldots, q_{n}\right)^{-1} \exp \left\{-\beta\left[-\bar{\mu}(n+1)+\frac{1}{2} \sum_{0}^{n} p_{i}^{2}\right.\right. \\
\left.\left.+U\left(q_{0}, \ldots, q_{n} \mid y\right)\right]\right\} d p_{0} \ldots d p_{n} \mu(d y),
\end{aligned}
$$

where $x \in \dot{x}_{\left\{q_{0}, \ldots, q_{n}\right\}}$ is of the form

$$
x=\left\{\left(q_{0}, p_{0}\right), \ldots,\left(q_{n}, p_{n}\right)\right\} \cup y,
$$

and $\varrho\left(q_{0}, \ldots, q_{n}\right)$ is the correlation function:

$$
\varrho\left(q_{0}, \ldots, q_{n}\right)=\left(e^{\beta \bar{\mu}}\left(\frac{2 \pi}{\beta}\right)^{d / 2}\right)^{n+1} \int_{,\left(q_{0} \ldots, q_{n} \uparrow\right)} \mu(d y) \exp \left\{-\beta U\left(q_{0}, \ldots, q_{n} \mid y\right)\right\} .
$$

On the quotient space $\mathscr{Y} / \boldsymbol{R}^{d}$ the $\sigma$-finite Borel measure $v$ is defined by the formula

$$
\begin{array}{r}
\int_{\boldsymbol{Y} / \mathbb{R}^{d}} f d v=\sum_{n=0}^{\infty} \frac{1}{(n+1) !} \int d q_{1} \ldots d q_{n} \varrho\left(q_{0}, \ldots, q_{n}\right) \int_{x_{\left\{q_{0}, q_{1}, \ldots, q_{n}\right\}}} \mu_{\left\{q_{0}, \ldots, q_{n}\right\}}(d x) \\
f\left(\left\{q_{0}, \ldots, q_{n}\right\}, x\right) .
\end{array}
$$

Since $\mu$ and $f$ are translation-invariant, this formula does not depend on the choice of the point $q_{0} \in \boldsymbol{R}^{d}$.

\section{Ergodic averages}

For translation-invariant $\mathrm{pq} f$ which as the function $f: \mathscr{Y} / \boldsymbol{R}^{d} \rightarrow \boldsymbol{R}^{1}$, is integrable with respect to the measure $v$, the phase-average, space-average and time-average can be defined.

(1) The phase-average, which is the real number, is defined as

$$
\lim _{V \rightarrow \infty} \frac{1}{|V|} \int_{i} f(x, V) \mu(d x)
$$

where $V \rightarrow \infty$ in Van-Hove sence. (Van-Hove convergence is defined in [5]). 
Phase-average theorem. For v-integrable $\mathrm{pq} f$ the phase-average exists and is equal to

$$
\int_{y / \mathbf{R}^{d}} f d v .
$$

Here, the only one condition about the interaction potential $U$ is existence of Gibbs measure $\mu$.

This theorem is a consequence of resuls in [1].

(2) The space-average (or thermodynamical limit), which is a translation-invariant $\mu$-integrable real function on the phase-space $\mathscr{X}$, is defined as

$$
\lim _{V \rightarrow \infty} \frac{1}{|V|} f(\cdot, V),
$$

where $V \rightarrow \infty$ in Van-Hove sense.

SPACE-AVERAGe theOREM [1]. For a v-integrable $\mathrm{pq} f$ the space-avervge exists in $L^{1}(., \mu)$-convergence.

If $V \nearrow \boldsymbol{R}^{d}$ and there exists a constant $C$ such that $|V-V|<C \cdot|V|$, where $V-V=\left\{v_{1}-v_{2}: v_{1}, v_{2} \in V\right\}$, then this limit exists $\mu$-always everywhere.

If Gibbs measure $\mu$ is ergodic, that means $\mu$ is a pure thermodynamical phase, then the space-average is constant and equals the phase-average.

Here, the only condition about the interaction potential $U$ is the existence of Gibbs measure $\mu$.

(3) The time-average, which is translation-invariant $p q$, that means is a real function on $: y / R^{d}$, is defined as

$$
\lim _{T \rightarrow \infty} \frac{1}{2 T} \int_{-T}^{T} S^{t} f d t
$$

Time-average may be studied if there exists a dynamics $\left\{S^{t}\right\}$, which can act on physical quantities. In order to define the $\mathrm{pq}^{t} f$ if a $\mathrm{pq} f$ is given we need to know the trajectories $(q, p)_{x}^{t}, t \in \boldsymbol{R}^{1}$, of particles $(q, p) \in x \in: X$.

Time-AVerage theorem [2]. Suppose that $\left\{S^{t}\right\}_{t \in R^{1}}$ is a system of automorphisms

$$
S^{t}: \mathscr{X}^{\prime \prime} \rightarrow \mathscr{X}^{\prime \prime}, \quad t \in \mathbf{R}^{1},
$$

where $. Y^{\prime \prime} \subset \mathscr{X}, \mu\left(\mathscr{X} \backslash \mathscr{X}^{\prime}\right)=0, S^{t} \cdot \mathscr{X}^{\prime \prime}=\mathscr{X}^{\prime \prime}, t \in R^{1}$, such that:

(1) $S^{t_{1}+t_{2}}=S^{t_{1}} S^{t_{2}}$

(2) for any particle $(q, p) \in x \in . Y^{\prime \prime}$ there exists a particle $\left(q_{x}^{t}, p_{x}^{t}\right) \in \boldsymbol{R}^{d} \times \boldsymbol{R}^{d}$ such that

$$
S^{t} x=\left\{\left(q_{x}^{t}, p_{x}^{t}\right):(q, p) \in x_{\}}^{\}},\right.
$$


(3) . $X^{\prime \prime}$ is translation-invariant and

$$
q_{x}^{t}+r=(q+r)_{(x+r)}^{t}, \quad r \in R^{d}, x \in \mathscr{Y}^{\prime \prime},
$$

(4) $R^{1} \times . X^{\prime \prime} \ni(t, x) \rightarrow S^{t} x \in . Y^{\prime \prime}$ is measurable,

(5) $\boldsymbol{R}^{1} \times\left(\mathscr{X}_{q} \cap \mathscr{X}^{\prime \prime}\right) \ni(t, x) \rightarrow q_{x}^{t} \in \boldsymbol{R}^{d}$ is measurable,

(6) $S^{t} \mu=\mu, t \in R^{1}$,

(7) for each $t \in R^{1}$

$$
\mu\left\{x \in \mathscr{X}^{\prime \prime}: \exists(q, p) \in x: \operatorname{dist}\left(q, q_{x}^{t}\right)=+\infty\right\}=0 .
$$

Then for $\mathrm{pq} f \in L^{p}\left(\mathscr{Y} / \boldsymbol{R}^{d}, v\right), p \geqslant 1$, the time-average exists $v$-always everywhere and is $L^{p}\left(y / \boldsymbol{R}^{d}, v\right)$-integrable.

If $p>1$, then time-average exists in $L^{p}\left(\mathscr{Y} / \boldsymbol{R}^{d}, v\right)$-convergence.

If $p=1$ and

$$
\operatorname{supp} f \subset \mathscr{Y}^{1} / \boldsymbol{R}^{d}, \quad \text { where } \mathscr{Y}^{1}=\{(A, x), \operatorname{Card} A=1\},
$$

then time-average exists in $L^{1}\left(y / R^{d}, v\right)$-convergence also. (The difference between cases $p>1$ and $p=1$ results from $v\left(\mathscr{Y} / \mathbb{R}^{d}\right)=\infty$.)

Here, the only one condition about the interaction potential $U$ is existence of Gibbs measure $\mu$.

The transferred dynamics $S^{t}$ on $\mathscr{Y} / R^{d}$ is not ergodic because subsets

$$
y^{n}=\{(A, x) \in \mathscr{Y}, \operatorname{Card} A=n\}, \quad n=1,2,
$$

are $S^{t}$-invariant.

If the transferred dynamics restricted to $: y^{n} / \mathbf{R}^{d}$ is ergodic and $n \geqslant 2$ then, for any $\mathrm{pq} f$ such that $\operatorname{supp} f \subset \mathscr{y}^{n} / \boldsymbol{R}^{d}$, the time-average, which is $L^{p}$ integrable and constant, equals zero $v$-a.e.

Sketch of the proof of the time-average theorem. $Y^{\prime}=\left\{(A, x) \in Y y: x \in: X^{\prime \prime}\right\}$ is translation-invariant and $y^{\prime} / \boldsymbol{R}^{d}$ has full measure $v$ :

$$
v\left(\mathscr{Y} / \boldsymbol{R}^{d} \backslash \mathscr{Y}^{\prime} / \boldsymbol{R}^{d}\right)=0 .
$$

The system of automorphisms $S^{t}: Y^{\prime \prime} \rightarrow: Y^{\prime \prime}, t \in \boldsymbol{R}^{1}$, can be transferred from $\mathscr{Y}^{\prime \prime}$ onto $9 Y^{\prime}$ and onto ${ }^{\prime \prime} Y^{\prime} / \boldsymbol{R}^{d}$ by the formula

$$
S^{t}(A, x)=\left(A_{x}^{t}, S^{t} x\right), \quad x \in \mathscr{Y}^{\prime \prime},
$$

where $A_{x}^{t}=\left\{q_{x}^{t}: q \in A\right\}$.

Conditions (4), (5) imply that

$$
R^{1} \times y^{\prime} \ni(t, A, x) \rightarrow\left(A_{x}^{t}, S^{t} x\right) \in y^{\prime}
$$

is measurable. 
Conditions (6), (7) show that the phase-average is constant in time:

$$
\int_{\mathscr{Q} / \mathbf{R}^{d}} S^{t} f d v=\int_{\mathscr{S} / \mathbf{R}^{d}} f d v, \quad t \in \boldsymbol{R}^{1},
$$

for any measurable $f$.

This is equivalent to

$$
S^{t} v=v, \quad t \in R^{1}
$$

So the transferred dynamics $S^{t}: \mathscr{Y} / \boldsymbol{R}^{d} \rightarrow \mathscr{Y} / \boldsymbol{R}^{d}$ satisfies the assumptions of the Birkhoff Ergodic Theorem. The assertion of the Birkhoff Ergodic Theorem for the transfered dynamics $\left\{S^{t}\right\}$ coincides with the thesis of time-average theorem, and this ends the proof.

\section{The central limit theorem}

After the space-average theorem, the next natural problem is the central limit theorem:

$$
\lim _{V \rightarrow \infty} \mu\left\{x \in \mathscr{X}: \frac{f(x, V)-\mathrm{Ef}(\cdot, V)}{[\operatorname{Var} f(\cdot, V)]^{1 / 2}} \in(a, b)\right\}=\frac{1}{(2 \pi)^{1 / 2}} \int_{a}^{b} e^{-t^{2} / 2} d t
$$

for every $a, b \in R^{1}$, which gives information on the speed of approximation of space-average when this limit is constant.

In probability theory the central limit theorems are studied usually for independent or weakly independent random variables.

There are also $p q$ 's which can be called independent: those which satisfy

$$
\begin{aligned}
& \mu_{A_{1}}\left\{x \in \mathscr{Y}_{A_{1}}: f\left(A_{1}, x\right) \in B_{1}\right\} \ldots \mu_{A_{n}}\left\{x \in \mathscr{Y}_{A_{n}}: f\left(A_{n}, x\right) \in B_{n}\right\}
\end{aligned}
$$

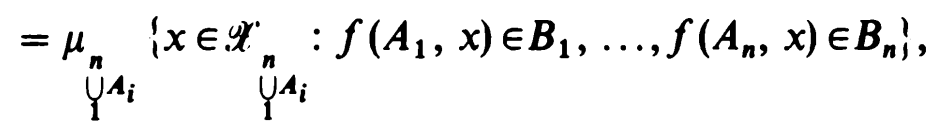

for pairwise disjoint finite $A_{i} \subset \boldsymbol{R}^{d}$ and any Borel $B_{i} \subset \boldsymbol{R}^{1}, i=1, \ldots, n$; $n=2, \ldots$

EXAMPLES OF INDEPENDENT pq's:

Ex 6. Point random field $f$ :

$$
\begin{gathered}
\operatorname{supp} f \subset \mathscr{Y}^{1}, \\
f(q, x)=h(p) \text { if }(q, p) \in x, \text { where } h: \boldsymbol{R}^{d} \rightarrow \boldsymbol{R}^{1} .
\end{gathered}
$$

This example includes Ex $1(h=1)$ and Ex $4\left(h(p)=p^{2} / 2\right)$.

Ex 3. Potential energy with null boundary condition: supp $f \subset \mathscr{y}^{2}$,

$$
f\left(\left\{q_{1}, q_{2}\right\}, x\right)=\varphi\left(q_{1}-q_{2}\right) \text {, }
$$

where $\varphi$ is the pair potential associated with the interaction potential. 
Ex 7. The observables for which Lanford proved the space-average theorem in [4]:

$$
\operatorname{supp} f \subset \bigcup_{k=1}^{n} y^{k}, \quad f\left(\left\{q_{1}, \ldots, q_{k}\right\}, x\right)=\varphi_{k}\left(q_{1}, \ldots, q_{k}\right),
$$

where $\varphi_{k}: R^{d k} \rightarrow R^{1}, k=1, \ldots, n$.

In Statistical Mechanics literature central limit theorems are proved for the following types of $p q$ 's: random fields and point random fields (discrete and continuous case respectively), potential energy with null boundary condition (see references in [3]).

In these proofs there are strong assumptions concerning the potential interactions of Gibbs measures.

From the viewpoint of the central limit theorem, random fields (discrete case) and point random fields (continuous case) differ in kind because point random fields are more random. In fact, in the discrete case it is assumed that in every point of lattice $Z^{d}$ a particle is situated with random spin (in the discrete case a particle is described by its position and spin). In the continuous case both position and momentum of a particle are random.

So, only in the continuous case it is possible that the central limit theorem holds for point random field represented by a function $h: R^{d} \rightarrow R^{1}$ but does not hold for point random field represented by the translating function $h+$ Const.

Central limit theorem [3]. If Gibbs measure $\mu$ satisties

$$
\begin{gathered}
\exists s<2, C>0, \forall V \text { bounded Borel } V \subset \boldsymbol{R}^{d}, \\
\operatorname{Var}\left(\operatorname{Card} x_{V}\right) \leqslant C \cdot|V|^{s},
\end{gathered}
$$

then central limit theorem holds for point random fields represented by a function $h: \boldsymbol{R}^{d} \rightarrow \boldsymbol{R}^{1}$, which have null mean and finite variation with respect to the Gauss measure $P$ on $R^{d}$

$$
P(d p)=(\beta / 2 \pi)^{1 / 2} \exp \left\{-\beta p^{2} / 2\right\} d p .
$$

Sketch of the proof. The proof is based on easily derivable formula

$$
\mathscr{L}(f(\cdot, V))=\sum_{n=0}^{\infty} \lambda_{V, n} \mathscr{L}(h)^{n}
$$

where

$$
f(x, V)=\sum_{(q, p) \in x, q \in V} h(p),
$$

$\mathscr{L}(f(\cdot, V))$ is the law (i.e. distribution) of random variable $f(\cdot, V)$ on the probability space $(\mathscr{X}, \mu)$, 
$\mathscr{L}(h)$ is the law of $h$ on the probability space $\left(\boldsymbol{R}^{d}, P\right)$, $\mathscr{L}(h)^{n}$ is the $n$th power of $\alpha(h)$ in sense of convolution, $\mathscr{L}(h)^{0}$ is the unit mass concentrated at zero, $\lambda_{V, n}=\mu\left\{x \in \mathscr{X} ;\right.$ Card $\left.x_{V}=n\right\}$.

The proof resolves itself into estimation of the distance $d_{1}$ between the measures

$$
\sum_{n=0}^{\infty} \lambda_{V, n} N(0, n / \varrho|V|) \quad \text { and } N(0,1),
$$

where the distance $d_{1}$ is defined by

$$
d_{1}\left(\mu_{1}, \mu_{2}\right)=\sup \left\{\left|\int f d\left(\mu_{1}-\mu_{2}\right)\right|: f \in C_{b}^{1},\|f\|_{\infty}+\left\|f^{\prime}\right\|_{\infty} \leqslant 1\right\},
$$

$N\left(0, \sigma^{2}\right)$ is the normal law on $R^{1}$ with expectation 0 and variance $\sigma^{2}$, $\varrho$ is the density: $\varrho|V|=\int_{,} \operatorname{Card} x_{V} d \mu$.

EXAMPLE of non-ergodic Gibbs measure such that the central limit theorem holds for a function $h$ with null mean, but does not hold for $h=1$.

Let $\mu_{1}, \mu_{2}$ be Gibbs measures satisfying the central limit theorem for $h$ $=1$ and such that

$$
\begin{gathered}
\int_{V} \operatorname{Card} x_{V} d \mu_{1}=\int_{Y} \operatorname{Card} x_{V} d \mu_{2}=\varrho|V|, \\
\lim _{V \rightarrow \infty}\left(\operatorname{Var}_{\mu_{i}} \operatorname{Card} x_{V}\right) /|V|=p_{i}, \quad i=1,2, p_{1} \neq p_{2} .
\end{gathered}
$$

Such measures exist.

Then the non-ergodic measure $\mu=\lambda_{1} \mu_{1}+\lambda_{2} \mu_{2}, 0<\lambda_{i}<1, \lambda_{1}+\lambda_{2}=1$, satisfies $(*)(=$ the assumption of the central limit theorem) but for $h=1$ we have

$$
\begin{aligned}
& \lim _{V \rightarrow \infty} \mathscr{L}\left(\frac{\operatorname{Card} x_{V}-\varrho|V|}{\left(\operatorname{Var} \operatorname{Card} x_{V}\right)^{1 / 2}}\right) \\
& \quad=\lambda_{1} N\left(0, \frac{p_{1}}{\lambda_{1} p_{1}+\lambda_{2} p_{2}}\right)+\lambda_{2} N\left(0, \frac{p_{2}}{\lambda_{1} p_{1}+\lambda_{2} p_{2}}\right) \neq N(0,1) .
\end{aligned}
$$

\section{References}

[1] K. Parczyk, Space-translation ergodic theorem for physical quantities, in infinite classical systems, Rep. Math. Phys. 24 (1986), 155-175.

[2] -, Time-average for physical quantities, in infinite classical systems, Rep. Math. Phys. 24 (1986), 265-292.

[3] K. Parczyk and T. Masłowski, Thermodynamical limit and central limit theorem for point random fields in nonergodic case, Rep. Math. Phys. 26 (1987).

[4] O. E. Lanford III, Entropy and equilibrium states in Classical Statistical Mechanics, in: Lecture Notes in Physics 20, Springer-Ver「ag, 1973, 1-113.

[5] D. Ruelle, Statistical mechanics: rigorous results. New York; Benjamin 1969. 